\title{
Atividades de vida e diagnósticos de enfermagem na população de rua
}

\author{
Life activities and nursing diagnoses of the street population \\ Actividades de vida y diagnósticos de enfermería en la población de la calle
}

\author{
Maria Aline Moreira Ximenes' @ ; Josiane da Silva Gomes’ @ ; Francisco Marcelo Leandro Cavalcante ${ }^{\prime \prime} \oplus^{\circledR}$;

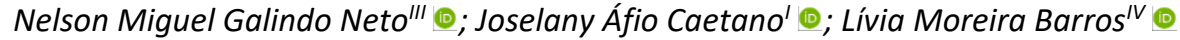

'Universidade Federal do Ceará, Fortaleza, CE, Brasil;"Universidade Estadual do Vale do Acaraú, Sobral, CE, Brasil;"'Instituto Federal de Educação, Ciência e
Tecnologia de Pernambuco, Pesqueira, PE, Brasil; "Vnniversidade da Integração Internacional da Lusofonia Afro-Brasileira, Fortaleza CE, Brasil

\section{RESUMO}

Objetivo: avaliar o desempenho das atividades de vida diárias e identificar diagnósticos de enfermagem de pessoas em situação de rua. Métodos: estudo transversal, realizado de abril a julho de 2017, com 52 pessoas. Para coleta de dados, foi utilizado instrumento fundamentado no modelo teórico de Roper-Logan e Tierney. Foram consideradas dependentes as atividades de vida com frequência igual ou superior a 40\%, para inferência dos diagnósticos utilizou-se referencial de Risner. Resultados: as atividades de vida dependentes foram: trabalho e distração $(92,3 \%)$, morte $(88,5 \%)$, sexualidade $(76,9 \%)$, sono $(71,2 \%)$, manter ambiente seguro (71,2\%), respirar (61,5\%), eliminar $(53,8 \%)$ e alimentar-se (51,9\%). Os diagnósticos mais frequentes: Disposição para comunicação melhorada (96\%), Desobstrução ineficaz das vias aéreas (94\%), Risco de contaminação (77\%) e Controle de impulsos ineficaz (73\%). Conclusão: evidenciou-se que pessoas em situação de rua possuem dependência de cuidados em atividades de vida diária relacionados a aspectos fisiológicos, sociais e emocionais.

Descritores: Teoria de Enfermagem; Diagnóstico de Enfermagem; Pessoas em Situação de Rua; Atividades Cotidianas

\section{ABSTRACT}

Objective: to evaluate performance of activities of daily living (ADLs) and to identify nursing diagnoses for people living on the streets. Methods: in this cross-sectional study of 52 people, data were collected between April and July 2017 using an instrument based on the Roper-Logan-Tierney theoretical model. ADLs with frequency of $40 \%$ or more were considered to be care-dependent; diagnoses were inferred using a Risner framework. Results: dependent ADLs were: working and playing $(92.3 \%)$, dying $(88.5 \%)$, expressing sexuality $(76.9 \%)$, sleeping $(71.2 \%)$, maintaining a safe environment $(71.2 \%)$, breathing $(61.5 \%)$, eliminating body wastes $(53.8 \%)$, and eating $(51.9 \%)$. The most frequent diagnoses were: improved communication disposition (96\%), ineffective airway clearance (94\%), contamination risk (77\%), and ineffective impulse control (73\%). Conclusion: people living on the streets were found to be dependent on care in physiological, social and emotional ADLs. Descriptors: Nursing Theory; Nursing Diagnosis; Homeless Persons; Activities of Daily Living.

\section{RESUMEN}

Objetivo: evaluar el desempeño de las actividades de la vida diaria e identificar diagnósticos de enfermería para personas que viven en la calle. Métodos: estudio transversal, realizado entre abril y julio de 2017, con 52 personas. Para la recolección de datos se utilizó un instrumento basado en el modelo teórico de Roper-Logan y Tierney. Se consideraron como dependientes las actividades de vida con una frecuencia igual o superior al 40\%. Para inferir los diagnósticos se utilizó el modelo referencial de Risner. Resultados: las actividades de la vida dependientes fueron: trabajo y distracción (92,3\%), muerte (88,5\%), sexualidad $(76,9 \%)$, sueño $(71,2 \%)$, mantener un ambiente seguro $(71,2 \%)$, respirar $(61,5 \%)$, eliminar $(53,8 \%)$ y alimentarse $(51,9 \%)$. Los diagnósticos más frecuentes: Mejora de la disposición comunicativa (96\%), Limpieza ineficaz de la vía aérea (94\%), Riesgo de contaminación (77\%) y Control ineficaz de los impulsos (73\%). Conclusión: se evidenció que las personas que viven en la calle son dependientes de cuidados en las actividades de la vida diaria relacionadas con los aspectos fisiológicos, sociales y emocionales. Descriptores: Teoría de Enfermería; Diagnóstico de Enfermería; Personas sin Hogar; Actividades Cotidianas.

\section{INTRODUÇÃO}

Pessoas em Situação de Rua (PsR) fazem parte de segmento populacional que vem aumentando consideravelmente nos últimos anos, o qual é caracterizado por condições de extrema pobreza. Estima-se que, em março de 2020, havia 221.869 PsR no Brasil, com maior concentração em municípios de grande porte ${ }^{1}$.

As PsR enfrentam, diariamente, situações desafiadoras como violência, privação de direitos e estigma social. Essa realidade tem impacto direto na saúde e na qualidade de vida dessas pessoas, pois possui maior probabilidade de resultar em condições crônicas, distúrbios mentais e transtornos por uso de substâncias psicoativas².

Neste cenário, a assistência a PsR apresenta-se como desafio aos profissionais e sistemas de saúde para garantir acessibilidade aos serviços e equidade das ações. Diante disso, conhecer o contexto dessa população, identificar suas particularidades e deficiências é uma das estratégias para ampliar a promoção da saúde desse grupo vulnerável ${ }^{3}$. Com isso, a enfermagem se destaca por participar da prestação de cuidados diretos a estes indivíduos nos diversos níveis de

Autor correspondente: Francisco Marcelo Leandro Cavalcante. E-mail: marceloleandrocavalcante98@hotmail.com

Editora Científica: Cristiane Helena Gallasch; Editora Associada: Thelma Spindola 
atenção à saúde, dispondo de ações organizadas e processos de trabalho com base na sistematização da assistência. Ao identificar as necessidades de saúde desta população a partir do processo de enfermagem, é possível estabelecer as ligações necessárias para a implementação de ações efetivas para promoção da saúde ${ }^{4}$.

Embora outros estudos tenham abordado questões relativas à saúde de PsR, há escassez de evidências apoiadas em referenciais teóricos e que apresentem os Diagnósticos de Enfermagem (DE) mais frequentes. Assim, este estudo proporciona esclarecimentos sobre realidade de PsR e suas necessidades de cuidado, o que poderá beneficiar diretamente a assistência a este público pela equipe multiprofissional, uma vez que, pode subsidiar o planejamento de intervenções individuais ou coletivas nos diversos níveis de atenção à saúde com intuito de favorecer o acesso à saúde desses indivíduos.

Ao considerar que os cuidados em enfermagem são influenciados pelo grau de dependência para Atividades de Vida Diárias (AVD) e que são implementados a partir de plano de cuidados, surgiu o seguinte questionamento: Qual o grau de desempenho das atividades de vida diárias e os diagnósticos de enfermagem da população em situação de rua? Desse modo, os objetivos desse estudo foram avaliar o desempenho das atividades de vida diárias e identificar diagnósticos de enfermagem de pessoas em situação de rua.

\section{REFERENCIAL TEÓRICO}

Optou-se em trabalhar com referencial teórico do modelo de AVD proposto por Roper-Logan e Tierney, o qual infere que é função específica do enfermeiro ajudar os indivíduos a evitar, aliviar, resolver ou, até mesmo, conviver com as situações de problemas ou desequilíbrios (reais ou potenciais) relacionados com suas AVD. O modelo propõe 12 Atividades de Vida: manter o ambiente seguro, respiração, alimentação, eliminação, comunicação, higiene pessoal e do vestuário, controle da temperatura corporal, trabalhar e divertir-se, mobilidade, exprimir a sexualidade, dormir e morrer ${ }^{5}$.

Com base no referencial de AVD é possível identificar os DE mais frequentes e com maior dependência de cuidados da enfermagem, o que favorece uma visão integral dos sujeitos e promove uma melhor identificação dos possíveis fenômenos de enfermagem. Dessa forma, o uso desse referencial torna-se uma ferramenta importante no direcionamento do plano de cuidado e tomada de decisões da equipe de enfermagem.

\section{MÉTOdOS}

Trata-se de estudo transversal, exploratório e descritivo com abordagem quantitativa, conduzido conforme as diretrizes da ferramenta Strengthening the Reporting of Observational Studies in Epidemiology (STROBE). Foi realizado de abril a julho de 2017, em Sobral-CE, em Centro de Referência Especializado a População em situação de Rua (Centro POP), que é unidade pública de referência no atendimento à população adulta em situação de rua.

A população do estudo foi representada por PsR que frequentavam o Centro POP, o que configurou a amostragem do tipo não probabilística intencional. Para cálculo amostral, foi solicitado, à coordenação do Centro POP, o número de indivíduos que frequentavam ativamente o local de estudo, totalizando 60 participantes. Assim, a amostra foi calculada a partir da fórmula para população finita com os seguintes parâmetros: N=60; nível de confiança do estudo de $95 \%(Z \alpha=1,96)$; erro amostral de 5\%; prevalência do evento de 50\%, por meio dos quais foi calculada amostra final de 52 participantes.

Os critérios de inclusão foram: ser usuário do Centro POP e possuir idade igual ou superior a 18 anos. Foram excluídas pessoas que não estavam em condições de participar da entrevista ou sob efeito de substâncias psicotrópicas.

Antes de ir a campo, as pesquisadoras receberam treinamento com profissional Doutor em Enfermagem, com expertise na temática a fim de garantir que todos aplicassem o instrumento de coleta de dados de forma padronizada. Para realizar as entrevistas, as pesquisadoras contaram com a colaboração dos funcionários do serviço, os quais, direcionavam os participantes para sala de entrevista após as refeições ofertadas no local. Neste momento, as pesquisadoras se apresentavam ao indivíduo e esclareciam os objetivos da pesquisa. Ao manifestar interesse em participar, eram analisados os critérios de inclusão e exclusão e, em seguida, assinado o Termo de Consentimento Livre e Esclarecido (TCLE).

Durante a coleta de dados, as entrevistas individuais duravam, em média, 15 minutos com aplicação de questionário semiestruturado dividido em duas partes em que a primeira contemplava a identificação dos participantes com dados socioeconômicos enquanto a segunda continha os questionamentos sobre 12 Atividades de Vida, baseado no modelo de Roper, Logan e Tierney ${ }^{5}$. Com base das respostas dos participantes, era possível avaliar se a AVD estava afetada ou não. Foram considerados como dependentes aquelas que apresentaram frequência igual ou superior a $40 \%$. A dependência significa que a AVD necessita de supervisão, intervenção ou ajuda para ser realizada ${ }^{5}$. No questionário também havia espaço para o pesquisador inferir os Diagnósticos de Enfermagem (DE) conforme a atividade de vida avaliada.

As respostas foram categorizadas e tabuladas no programa Microsoft Excel e, em seguida, exportadas para o Statistical Package for the Social Sciences (SPSS) versão 22.0. Os dados foram apresentados em frequências absolutas e relativas. Para inferência dos $\mathrm{DE}$, foi utilizado o referencial de Risner ${ }^{6}$ que estabelece as etapas de análise e síntese. Após 
a identificação das necessidades apresentadas pelos pacientes, os diagnósticos foram elencados de acordo com a taxonomia II da NANDA-I, considerou-se a definição do diagnóstico, fatores relacionados e características definidoras? ${ }^{7}$.

O estudo foi desenvolvido de acordo com a Resolução 466/2012 do Conselho Nacional de Saúde do Brasil, com a aprovação do Comitê de Ética em Pesquisa da instituição proponente.

\section{RESULTADOS}

Na Tabela 1, é possível visualizar o perfil socioeconômico dos 52 participantes do estudo, cuja média de idade de foi de $37,62( \pm 9,8)$.

TABELA 1: Perfil socioeconômico das pessoas

em situação de rua. Sobral, CE, Brasil, 2017.

\begin{tabular}{|c|c|c|}
\hline Variáveis & $\mathbf{N}$ & $\%$ \\
\hline \multicolumn{3}{|l|}{ Sexo } \\
\hline Masculino & 46 & 88,5 \\
\hline Feminino & 6 & 11,5 \\
\hline \multicolumn{3}{|l|}{ Estado Civil } \\
\hline Casado & 7 & 13,5 \\
\hline Divorciado & 13 & 25,0 \\
\hline União Estável & 4 & 7,7 \\
\hline Solteiro & 27 & 51,9 \\
\hline Viúvo & 1 & 1,9 \\
\hline \multicolumn{3}{|l|}{ Filhos } \\
\hline Sim & 38 & 73,07 \\
\hline Não & 14 & 26,93 \\
\hline \multicolumn{3}{|l|}{ Religião } \\
\hline Católica & 26 & 50,0 \\
\hline Evangélica & 16 & 30,8 \\
\hline Ateu & 9 & 17,3 \\
\hline Testemunha de Jeová & 1 & 1,9 \\
\hline \multicolumn{3}{|l|}{ Escolaridade } \\
\hline 0 a 8 anos & 28 & 53,84 \\
\hline 9 a 11 anos & 21 & 40,39 \\
\hline Acima de 12 anos & 3 & 5,77 \\
\hline \multicolumn{3}{|l|}{ Vínculo Empregatício } \\
\hline Ativo & 10 & 19,2 \\
\hline Inativo & 42 & 80,8 \\
\hline \multicolumn{3}{|l|}{ Renda } \\
\hline Até 1 salário mínimo & 49 & 94,23 \\
\hline Até 2 salários mínimos & 3 & 5,77 \\
\hline \multicolumn{3}{|l|}{ Procedência } \\
\hline Fortaleza & 8 & 15,39 \\
\hline Sobral & 19 & 36,54 \\
\hline Macrorregião de Sobral & 16 & 30,77 \\
\hline Outros estados & 9 & 17,30 \\
\hline
\end{tabular}

Fonte: Dados da pesquisa.

Entre os fatores que os motivaram a viver nas ruas, destacam-se conflitos familiares ( $n=34,65,39 \%)$, uso abusivo de drogas ( $n=12,23,07 \%)$ e desemprego $(n=6,11,54 \%)$. Ao avaliar o tempo que se encontravam em situação de rua, houve predomínio de vivência nas ruas de até seis meses $(38,47 \%$ ) e acima de quatro anos $(28,84 \%)$. Foi constatado que $47(91,40 \%)$ participantes desejavam mudar sua situação socioeconômica e sair das ruas, o mesmo percentual afirmou satisfação com atendimento dos profissionais do Centro POP. Com relação ao atendimento dos serviços de saúde, 36 $(69,20 \%)$ participantes avaliaram como bom e resolutivo.

A Tabela 2 apresenta as AVD de acordo com a dependência para sua realização. 
TABELA 2: Classificação das Atividades de Vida Diária dependentes e independentes de pessoas em situação de rua. Sobral, CE, Brasil, 2017.

\begin{tabular}{lcc}
\hline Atividades & Dependente & Independente \\
\cline { 2 - 3 } & $\mathbf{n}(\%)$ & $\mathbf{n}(\%)$ \\
\hline Manter ambiente seguro & $37(71,2)$ & $15(28,8)$ \\
Comunicar & $22(42,3)$ & $30(57,7)$ \\
Respirar & $32(61,5)$ & $20(38,5)$ \\
Alimentar & $27(51,9)$ & $25(48,1)$ \\
Eliminar & $28(53,8)$ & $24(46,2)$ \\
Higiene e Vestuário & $19(36,5)$ & $33(63,5)$ \\
Temperatura & $10(19,2)$ & $42(80,8)$ \\
Mobilidade & $17(32,7)$ & $35(67,3)$ \\
Trabalho e Distração & $48(92,3)$ & $04(7,7)$ \\
Sexualidade & $40(76,9)$ & $12(23,1)$ \\
Sono & $37(71,2)$ & $15(28,8)$ \\
Morte & $46(88,5)$ & $06(11,5)$ \\
\hline
\end{tabular}

Fonte: Dados da pesquisa.

As AVD que apresentaram maior dependência foram: trabalho e distração, morte, manutenção do ambiente seguro, sono, sexualidade, alimentação, eliminação e respiração. Já os DE são apresentados na Tabela 3, divididos conforme as AVD.

TABELA 3: Diagnósticos de enfermagem da NANDA presentes em pessoas em situação de rua. Sobral, CE, Brasil, 2017.

\begin{tabular}{|c|c|c|c|}
\hline \multicolumn{2}{|r|}{ Diagnósticos de Enfermagem } & \multirow{2}{*}{$\begin{array}{l}\mathbf{n} \\
28\end{array}$} & \multirow{2}{*}{$\begin{array}{l}\% \\
53\end{array}$} \\
\hline Manutenção de um ambiente seguro & (00174) Risco de dignidade humana comprometida & & \\
\hline & (00043) Proteção ineficaz & 17 & 32 \\
\hline & (00171) Disposição para religiosidade melhorada & 4 & 7 \\
\hline & (00138) Risco de violência direcionada a outros & 2 & 3 \\
\hline \multirow[t]{3}{*}{ Comunicação } & (00157) Disposição para comunicação melhorada & 50 & 96 \\
\hline & (00058) Risco de vínculo prejudicado & 31 & 59 \\
\hline & (00052) Interação social prejudicada & 15 & 28 \\
\hline \multirow[t]{2}{*}{ Respiração } & (00031) Desobstrução ineficaz das vias aéreas & 49 & 94 \\
\hline & (00032) Padrão respiratório ineficaz & 15 & 28 \\
\hline \multirow[t]{3}{*}{ Alimentação } & (00180) Risco de contaminação & 40 & 77 \\
\hline & (00002) Nutrição desequilibrada: menor do que as necessidades corporais & 31 & 60 \\
\hline & (00163) Disposição para nutrição melhorada & 15 & 29 \\
\hline \multirow[t]{2}{*}{ Eliminação } & (00016) Eliminação urinária prejudicada & 24 & 46 \\
\hline & (00011) Constipação & 25 & 48 \\
\hline \multirow[t]{2}{*}{ Higiene e vestuário } & (00247) Risco de integridade da membrana mucosa oral prejudicada & 13 & 25 \\
\hline & (00214) Conforto prejudicado & 10 & 19 \\
\hline \multirow[t]{7}{*}{ Mobilidade Trabalhar e distração } & (00085) Mobilidade física prejudicada & 13 & 25 \\
\hline & (00222) Controle de impulsos ineficaz & 38 & 73 \\
\hline & (00052) Interação social prejudicada & 29 & 56 \\
\hline & (00188) Comportamento de saúde propenso a risco & 22 & 42 \\
\hline & (00097) Envolvimento em atividades de recreação diminuído & 18 & 35 \\
\hline & (00207) Disposição para relacionamento melhorado & 17 & 33 \\
\hline & (00054) Risco de Solidão & 6 & 11 \\
\hline \multirow[t]{2}{*}{ Sexualidade } & (00065) Padrão de sexualidade ineficaz & 29 & 69 \\
\hline & (00059) Disfunção Sexual & 18 & 35 \\
\hline \multirow[t]{5}{*}{ Sono } & (00198) Distúrbio no padrão de sono & 28 & 54 \\
\hline & (00214) Conforto prejudicado & 26 & 50 \\
\hline & (00136) Pesar & 27 & 52 \\
\hline & (00147) Ansiedade relacionada à morte & 21 & 40 \\
\hline & (00150) Risco de Suicídio & 19 & 36 \\
\hline
\end{tabular}

Fonte: Dados da pesquisa. 
Foi possível identificar como DE frequentes, os problemas reais: Disposição para comunicação melhorada ( $96 \%)$, Desobstrução ineficaz das vias aéreas (94\%) e Controle de impulsos ineficaz (73\%). Dentre os DE de risco, destacou-se Risco de contaminação (77\%). Sete DE foram de risco, 19 representaram problemas reais e quatro relacionados à promoção da saúde.

\section{DISCUSSÃO}

Neste estudo, foi possível identificar perfil predominante de homens, solteiros com média de idade de $37,62( \pm 9,8)$ anos e escolaridade entre zero e oito anos de estudo. Esses dados assemelham-se aos resultados de estudos realizados em Pernambuco, São Paulo e Porto Alegre e em outros países como Estados Unidos e Etiópia, cujas amostras foram representadas majoritariamente por homens, solteiros com baixa escolaridade que vivem em centros urbanos em extrema pobreza ${ }^{2,4,8,9,10}$. Assim, cabe à enfermagem atentar para questões que permeiam a saúde do homem em meio a PsR, uma vez que esta é constituída, em sua maioria, pelo público masculino.

A maioria dos indivíduos relatou crenças espirituais com apego à fé e ao ser superior para enfrentar as adversidades encontradas no cotidiano. $\mathrm{O}$ apego a fé gera fortalecimento, esperança e felicidade tendo em vista que essa ação reforça os sentimentos positivos que a pessoa tem de si mesma. Crenças espirituais e esperança são mecanismos de enfrentamento de fundamental importância e que podem contribuir para o autocuidado das PsR ${ }^{4}$. Esses dados também revelam a importância da avaliação espiritual em futuras pesquisas, para avaliar a influência desses fatores no enfretamento do processo de viver nas ruas.

Verificou-se também que o tempo médio de moradia nas ruas foi entre seis meses e quatro anos e os principais fatores motivadores foram conflitos familiares, uso abusivo de drogas e desemprego. Estudo realizado na Colômbia com 60 PsR evidenciou que as principais causas desta condição se relacionam a problemas econômicos e contextos vulneráveis como conflitos familiares, situações de abuso, maus-tratos, abandono, vícios e violência ${ }^{11}$, o que corrobora com achados do presente estudo. Assim, os profissionais de saúde devem estar atentos aos determinantes sociais que permeiam a realidade de cada PsR, para buscar garantir assistência intersetorial, interprofissional e integral a fim de facilitar a implementação de intervenções eficazes que favoreçam a mitigação dos possíveis conflitos existentes na vida desses indivíduos e promovam saúde e bem-estar.

O uso de drogas também é uma das principais razões pelas quais indivíduos se encontram em situação de rua. Essa prática está diretamente associada à violência, haja vista que a necessidade de alimentar o vício acarreta diversos problemas como roubos e prostituição. Estudo realizado em Uganda, com 346 jovens usuários de drogas, incluindo PsR, identificou que $46 \%$ destes estiveram envolvidos com situações de violência em que foram alvo delas ou praticaram as ações violentas ${ }^{12}$.

Com isso, destaca-se que o envolvimento com drogas e violência expõe o indivíduo a situações de vulnerabilidade e aumenta o risco de morte. É pertinente a implementação de ações educativas voltadas a redução de danos e que visem orientar sobre os riscos relacionados ao uso abusivo de álcool e outras drogas, bem como estratégias de autocuidado que podem reduzir os impactos físicos e psicológicos dessas substâncias.

Ressalta-se, ainda, que o uso de drogas favorece comportamento suicida entre as PsR ${ }^{13}$. Dessa forma, os aspectos que envolvem a saúde mental e a situação de dependência química são relevantes no plano de cuidados da enfermagem, no qual deve-se dedicar atenção para a detecção de transtornos mentais comuns e auxílio no acompanhamento das pessoas acometidas por meio de grupos de apoio que podem ser implantados em Unidades Básicas de Saúde ou em Centros de Apoio Psicossocial ${ }^{14}$.

Ao analisar o grau de desempenho na realização das AVD, verificou-se que a maior necessidade de cuidado de enfermagem relacionadas aos DE reais esteve ligada às intervenções que melhorem o desempenho de funções fisiológicas como alimentar-se, eliminar, respirar e dormir, além de atividades básicas como manter ambiente seguro, exprimir sexualidade, trabalho e distração.

$\mathrm{Na}$ atividade de vida alimentar-se foi encontrado o DE Nutrição desequilibrada: menor do que as necessidades corporais, o qual é definido como ingestão insuficiente de nutrientes para satisfazer às necessidades metabólicas ${ }^{7}$. Neste estudo, identificou-se que o consumo de alimentos se restringe às refeições ofertadas no Centro POP ou por meio de doações, as quais não ocorrem com frequência. Associado à essa realidade, as PsRs apresentam fatores de risco que potencializam a desnutrição como alcoolismo, uso de drogas, doenças físicas e mentais, o que alerta para necessidade de políticas públicas que viabilizem a melhoria da nutrição dessa população vulnerável ${ }^{15}$.

Pesquisa qualitativa com 122 PsR na Bahia corrobora com esses achados ao inferir que PsR não possuem acesso adequado à alimentação saudável para garantia da necessidade básica diária que é alimentar-se. As refeições disponibilizadas em instituições como o Centro POP ocorrem somente de segunda a sexta. Assim, nos finais de semana, não há fornecimento de alimentação para esses indivíduos, o que favorece a fome, desnutrição, desesperança e tristeza 
diante dessa situação de vida ${ }^{16}$. Diante disso, é relevante garantir o acesso da população de rua à alimentação saudável, visto que isso viabilizará a manutenção da alimentação diária rica em nutrientes para esses indivíduos.

Vale ressaltar, ainda, que a atividade de vida alimentar-se também sofre influência da saúde bucal. Estudo realizado em Goiânia-GO com 120 indivíduos que vivem nas ruas evidenciou que 59,5\% dos participantes revelaram dificuldade para comer devido à ausência de dentes ou presença de problemas dentários ${ }^{17}$. Cabe ao enfermeiro, como educador em saúde, orientar a busca dos serviços da atenção primária para tratamento odontológico e realizar orientações sobre higiene bucal.

Com relação à atividade eliminar, um dos DE mais prevalentes foi a Constipação que é definido pela diminuição na frequência normal de evacuação, acompanhada por eliminação difícil ou incompleta de fezes e/ou eliminação de fezes excessivamente duras e secas ${ }^{7}$. Neste estudo, os principais fatores relacionados são falta de privacidade para evacuar ou local inapropriado, ingestão hídrica inadequada e refeições irregulares.

Estudo realizado em Sobral-CE com 52 PsR mostrou que os participantes passavam cerca de cinco dias sem evacuar, e os autores associaram a constipação à má alimentação e baixa disponibilidade banheiros públicos ${ }^{18}$. Dessa forma, é preciso garantir que a PsR tenha acesso a locais públicos, onde possam realizar suas necessidades de eliminações adequadamente, seja por meio de Centros de referência ou banheiros públicos, destinados a este fim. Para isso, faz-se necessário atuação conjunta entre os setores da saúde e governamentais para desenvolvimento urbano voltado para a população de rua.

No que se refere a respiração, verificou-se que a maioria dos participantes apresentou o DE Desobstrução ineficaz de vias aéreas, que está relacionada à incapacidade de eliminar secreções ou obstruções do trato respiratório para manter a via aérea desobstruída evidenciada pelo relato de tosse ineficaz, dispneia e roncos ${ }^{7}$.

Estudo realizado em Boston analisou os fatores relacionados aos problemas respiratórios em PsR e identificou que fatores comportamentais como beber cinco ou mais vezes por semana está associado a maior risco de contrair infecções respiratórias. Ademais, destacou-se que a transitoriedade aumentada na localização do sono, definida como dormir em mais de um local na última semana, foi associada ao aumento de $40 \%$ na chance de colonização nasal. O banho diário foi associado a redução de $70 \%$ na chance de desenvolver problemas respiratórios ${ }^{19}$.

Evidencia-se que políticas públicas que possibilitem a disponibilização de abrigos comunitários com banheiros teriam impacto direto na redução de problemas respiratórios entre pessoas que vivem nas ruas, além de contribuir para redução da marginalização e estigmatização dessa população ao assegurar ambiente saudável para atividades de vida básicas como manutenção do sono e higiene.

Em relação à atividade dormir, observou-se, neste estudo, que existe dependência do cuidado de enfermagem, uma vez que muitos participantes relataram fazer uso excessivo de álcool para favorecer o sono, além de queixas como baixa qualidade do sono relacionado ao ambiente, fadiga e sonolência diurna. Assim, houve predomínio, entre os participantes deste estudo, do DE Distúrbio no Padrão de Sono, que é definido como: despertares com tempo limitado em razão de fatores externos ${ }^{7}$.

A inadequação do sono pode estar associada com efeitos negativos no estado de saúde com reflexo no humor, cognição e energia. Estudo realizado em João Pessoa-PB com 49 pessoas em situação de rua mostrou que 61,2\% dos participantes dormem mal devido à privação de ambientes que favoreçam o sono. Os participantes afirmaram dormir, muitas vezes, em praças e calçadas sob os efeitos de frio, vento e chuva ${ }^{14}$.

Para essa população, o sono pode ser interrompido por diversos fatores como condições ambientais relacionadas ao mau tempo, já que, o sono geralmente ocorre em áreas públicas abertas, além da necessidade de manter-se vigilante enquanto dorme para se proteger-se contra roubo, falta de privacidade, além das desconfortáveis condições de sono ${ }^{20}$.

No que se refere à atividade manter ambiente seguro, os participantes relataram episódios de violência e restrição de um lar e, a partir dessas queixas dos entrevistados, foi possível inferir a presença dos DE como Ansiedade relacionada à morte, Pesar e Conforto prejudicado relacionados a sensação de insegurança vivida por estes.

Estudo qualitativo realizado com cinco PsR no Distrito Federal revelou que esses indivíduos apresentam constante sentimento de medo ao viver nas ruas por não terem conhecimento sobre como seria o dia seguinte, se estariam vivos e se teriam algo para comer, além do receio da vivência diária de situações de violência ${ }^{21}$. Nessa perspectiva, evidenciase a necessidade de suporte social e terapêutico para esses indivíduos, a fim de promover conforto adequado e alívio de sentimentos negativos relacionados à vivência nas ruas. Para este momento, torna-se pertinente que o enfermeiro faça uso do processo de enfermagem para identificação das necessidades básicas de cuidado de cada indivíduo.

Em relação a atividade de vida sexualidade, o DE principal foi Padrão de sexualidade ineficaz definido por expressões de preocupação quanto à própria sexualidade ${ }^{7}$. Apesar de quase todos os participantes terem referido manter vida sexual ativa, muitos afirmaram não utilizar preservativos. Dentre as PsR que faziam uso de métodos 
contraceptivos, o preservativo masculino foi relatado como o principal método escolhido para prevenção de Infecções Sexualmente Transmissíveis (ISTs).

Contrastando com esses achados, estudo revela que o cuidado de si a partir de ações preventivas esteve no discurso de PsR entrevistados na Bahia, os quais afirmaram ser importante usar preservativos e fazer exames de rotina para ISTs ${ }^{16}$. Outrossim, estudo realizado no Brasil com 209 pessoas em situação de rua identificou prevalência de $27,8 \%$ de casos de infecção pelo vírus da imunodeficiência humana, 24,9\% por sífilis e 1\% por hepatite B, o que sinaliza para a importância da disponibilização e realização de consultas e exames preventivos anualmente para essa população, como também da disseminação de conhecimento sobre IST por meio de ações educativas ${ }^{22}$.

$\mathrm{Na}$ atividade de vida trabalho e distração foi identificado o DE Controle de impulsos ineficaz que é definido como padrão de reações rápidas e não planejadas a estímulos internos ou externos, sem levar em conta as consequências negativas dessas reações ao indivíduo impulsivo ou aos outros ${ }^{7}$. A situação de viver nas ruas contribui para estigmatização desses indivíduos como agressivos e usuários de drogas ${ }^{23}$, o que prejudica a participação e interação social, assim como a inclusão em ambientes laborais.

O estigma social e identidade marginalizada pela condição de viver nas ruas podem contribuir para sentimento de incapacidade desses indivíduos, que afeta diretamente a sua relação com o trabalho e socialização ${ }^{24}$. Assim, as dificuldades de socializar-se relacionadas ao trabalho, desemprego ou vínculos frágeis compõem o conjunto de fatores que os levam e os mantém em situação de rua.

São quase inexistentes oportunidades de trabalho formal para pessoas sem endereço fixo, mesmo que tenha competência para a função. Revisão Sistemática apontou que é comum PsR sobreviverem vigiando carros, catando lixo ou pedindo dinheiro nas ruas ${ }^{25}$. Destaca-se, então, a necessidade de envolvimento de setores governamentais, jurídico e industrial para criação de oportunidades de emprego e renda para PsR, o que contribuirá para redução do número de indivíduos que permanecem nas ruas devido ao aumento das possibilidades de melhores condições de vida.

É preciso, ainda, fomentar e ampliar o acesso aos centros de atendimento, tratamento, reabilitação e apoio aos transtornos mentais e ao uso de substâncias, com maior integração entre os diversos níveis de atenção à saúde mental, com vista a oportunizar o atendimento das necessidades dos sujeitos em situação de rua para promover a redução de danos e facilitar sua reinserção social ${ }^{10}$.

Assim, acredita-se que este estudo fornece aporte fundamentado para prática da enfermagem e para outros profissionais e pesquisadores com interesse pelo tema proposto. Além disso, traz consigo a aplicação de um modelo teórico próprio da enfermagem na PsR e propõe resultados que representam este grupo populacional. Logo, poderá servir como base para intervenções que busquem a prestação de cuidados efetivos, humanizados e qualificados que contemplem as particularidades dessa população vulnerável.

\section{Limitações do estudo}

Como limitações do estudo, destaca-se a composição amostral, por ter sido composta somente por pessoas em situação de rua que frequentavam o Centro POP. Assim, os achados podem não corresponder à realidade das pessoas que não são atendidas por esses serviços. A escassez de produções fundamentadas em referenciais teóricos, voltadas a esse grupo populacional, também configurou uma limitação.

\section{CONCLUSÃO}

Este estudo evidencia que PsR possuem dependência de cuidados em atividades de vida diária que se relacionam com diagnósticos de enfermagem reais, os quais refletem problemas fisiológicos, sociais e emocionais. Esses achados traduzem a necessidade de intervenções imediatas e a longo prazo, voltadas a garantia do acesso a serviços de saúde e assistência multiprofissional.

Os profissionais de enfermagem, como gerenciadores dos casos, devem explorar novos meios de garantir cuidados básicos e preventivos a PsR. Logo, há necessidade de integração entre serviços de saúde e programas de apoio disponíveis na comunidade. Estratégias direcionadas a construção de plano de cuidados individualizado e acompanhamento longitudinal podem beneficiar a oferta de informações, adesão a tratamentos e melhoria da qualidade de vida dessa população.

Os resultados indicam que, embora existam políticas públicas para PsR, o acesso aos serviços e a adesão as práticas de saúde continuam limitados. Portanto, ressalta-se a importância do fortalecimento e integração dos serviços assistenciais, de gestão, ensino e pesquisa, com o objetivo de ampliar espaços de discussão sobre formas efetivas para reduzir iniquidades sociais nesse grupo populacional e identificar maneiras efetivas de reinserção social e reconstrução permanente da vida dessas pessoas. 


\section{REFERÊNCIAS}

1. Natalino MAC. Estimativa da população em situação de rua no Brasil (setembro de 2012 a março de 2020). [Internet]. Brasília: Instituto de Pesquisa Econômica Aplicada; 2020 [cited 2021 Jan 04]. 36 p. Available from: https://www.ipea.gov.br/portal/images/stories/PDFs/nota_tecnica/200612_nt_disoc_n_73.pdf.

2. Landefeld JC, Miaskowski C, Tieu L, Ponath C, Lee CT, Guzman D, et al. Characteristics and Factors Associated With Pain in Older Homeless Individuals: results from the health outcomes in people experiencing homelessness in older middle age (hope home) study. J Pain [Internet]. 2017 [cited 2020 Apr 6]; 18(9):1036-45. DOI: https://doi.org/10.1016/j.jpain.2017.03.011.

3. Brandão MGS, Aragão C, Ximenes MA, Caetano J. Self-concept, depression and anxiety of street people. Rev Enferm Atual In Derme [Internet]. 2019 [cited 2020 Apr 7]; 90(28):1-6. Available from: https://revistaenfermagematual.com/index.php/revista/article/view/404/554.

4. Silva FP, Paiva FAS, Guedes CP, Frazão IS, Vasconcelos SC, Lima MDC. Nursing Diagnoses of the Homeless Population in Light of Self-care Theory. Arch Psychiatr Nurs [Internet]. 2018 [cited 2020 Apr 6]; 32(3):425-31. DOI: https://doi.org/10.1016/j.apnu.2017.12.009.

5. Roper N, Logan W, Tierney A. The Roper, Logan, Tierney Modelo of Nursing based on activities of living. London: Churchill Livingstone, 2000. $216 \mathrm{p}$.

6. Risner PB. Diagnosis: analysis and synthesis of data. In: Griffith-Kenney JW, Christensen PJ. Nursing Process application of theories, frameworks, and models. Saint Louis: The C.V. Mosby Company; 1986. 429 p.

7. NANDA International. Diagnósticos de enfermagem da NANDA-I: definições e classificação 2018-2020. 11a ed. Tradução deRegina Machado Garcez. Porto Alegre: Artmed; 2018.

8. Lussi IAO, Ricci TE, Pinho RJ. Perceptions on work: analysis of conceptions of homeless people. Cad Bras Ter Ocup [Internet]. 2017 [cited 2020 Apr 8]; 25(4):779-93. DOI: http://dx.doi.org/10.4322/2526-8910.ctoA00942.

9. Pimenta MM. Pessoas em situação de rua em Porto Alegre: Processos de estigmatização e invisibilidade social. Rev Ciênc Soc [Internet]. 2019 [cited 2020 Apr 8]; 19(1):82-104. DOI: https://doi.org/10.15448/1984-7289.2019.1.30905.

10. Ayano G, Assefa D, Haile K, Chaka A, Solomon H, Hagos P, et al. Mental, neurologic, and substance use (MNS) disorders among street homeless people in Ethiopia. Ann Gen Psychiatry [Internet]. 2017 [cited 2020 Apr 09]; 16(16):1-6. DOI: https://doi.org/10.1186/s12991-017-0163-1.

11. Baptista CM, Herrera GE, Piedrahíta LZ. Entre el hogar y el asfalto: relatos y experiencia de vida de habitantes en condición de calle. Rev Lasallista Investig [Internet]. 2017 [cited 2020 Apr 10]; 14(2):65-72. DOI: https://doi.org/10.22507/rli.v14n2a6.

12. Swahn MH, Culbreth R, Tumwesigye NM, Topalli V, Wright E, Kasirye R. Problem Drinking, Alcohol-Related Violence, and Homelessness among Youth Living in the Slums of Kampala, Uganda. Int J Environ Res Public Health [Internet]. 2018 [cited 2020 11]; 15(1061):1-13. DOI: https://doi.org/10.3390/ijerph15061061.

13. Castro RAS, Padilha ÉB, Dias CM, Botti NCL. Vulnerabilities of the homeless population to suicide behavior. Rev enferm UFPE on line [Internet]. 2019 [cited 2020 Apr 11]; 13(2):431-7. Available from: https://periodicos.ufpe.br/revistas/revistaenfermagem/article/download/237023/31353.

14. Patrício ACFA, Silva RAR, Araújo RF, Silva RF, Nascimento GTS, Rodrigues TDB, et al. Common mental disorders and resilience in homeless persons. Rev Bras Enferm [Internet]. 2019 [cited 2020 Apr 12]; 72(6):1526-33. DOI: http://dx.doi.org/10.1590/00347167-2018-0541.

15. Koh KA, Bharel M, Henderson DC. Nutrition for homeless populations: shelters and soup kitchens as opportunities for intervention. Public Health Nutr [Internet]. 2016 [cited 2020 Apr 12]; 19(7):1312-34. DOI: https://doi.org/10.1017/s1368980015002682.

16. Silva DO, Oliveira JF, Porcino C, Gomes AMT, Suto CSS, Carvalho ESS. Homeless people's social representations about self-care. Rev Bras Enferm [Internet]. 2020 [cited 2020 Apr 15]; 73(2):e20180956. DOI: https://doi.org/10.1590/0034-7167-2018-0956.

17. Lawder JAC, Matos MA, Souza JB, Freire MCM. Impact of oral condition on the quality of life of homeless people. Rev Saúde Pública [Internet]. 2019 [cited 2020 Apr 18]; 53:22. DOI: https://doi.org/10.11606/s1518-8787.2019053000718.

18. Gomes JS, Ximenes MAM, Brandão MGSA, Brito OD, Barros LM. Application of the Roper, Logan and Tierney model to homeless people. Rev Pesqui (Univ Fed Estado Rio J., Online) [Internet]. 2020 [cited Apr 18]; 12:238-45. Available from: http://www.seer.unirio.br/index.php/cuidadofundamental/article/view/8305.

19. Leibler JH, León C, Cardoso LJP, Morris JC, Miller NS, Nguyen DD, et al. Prevalence and risk factors for MRSA nasal colonization among persons experiencing homelessness in Boston, MA. J Med Microbiol [Internet]. 2017 [cited 2020 Apr 20]; 66(8):1183-8. DOI: https://doi.org/10.1099/jmm.0.000552.

20. Chang HL, Fisher FD, Reitzel LR, Kendzor DE, Nguyen MA, Businelle MS. Subjective sleep inadequacy and self-rated health among homeless adults. Am J Health Behav [Internet]. 2015 [cited 2020 Apr 20]; 39(1):14-21. DOI: https://doi.org/10.5993/ajhb.39.1.2.

21. Mota FO, Fonseca RMAM, Santos JE, Gallassi AD. Aspects of the whole care for people in street followed by health and social assistance services: a look at and for occupational therapy. Cad Bras Ter Ocup [Internet]. 2019 [cited 2020 Apr 21]; 27(4):80616. DOI: https://doi.org/10.4322/2526-8910.ctoao1809.

22. Matos MA, Caetano KAA, Teles SA, Pinheiro RS, Carvalho PMRS, Rosa LRC, et al. Evaluating the prevalence and knowledge of sexually transmitted infection among homeless persons in central Brazil. Sex Transm Infect [Internet]. 2017 [cited 2020 Jun 22]; 93(Suppl 2). DOI http://dx.doi.org/10.1136/sextrans-2017-053264.379.

23. Mendes KT, Ronzani TM, Paiva FS. Homeless population, vulnerabilities and drugs: a systematic review. Psicol Soc [Internet]. 2019 [cited 2020 Jun 22]; 31:e169056. DOI: https://doi.org/10.1590/1807-0310/2019v31169056. 
24. Sanders B. Brown B. 'I was all on my own': experiences of loneliness and isolation amongst homeless people [Internet]. London: Crisis; 2015 [cited 2019 Oct 20]. 29 p. Available from: https://www.crisis.org.uk/media/20504/crisis_i_was_all_on_my_own_2016.pdf.

25. Sicari AA, Zanella AV. Pessoas em Situação de Rua no Brasil: Revisão Sistemática. Psicol cienc prof [Internet]. 2018 [cited 2020 Oct 20]; 38(4):662-79. DOI: https://doi.org/10.1590/1982-3703003292017. 